\title{
Heartbeat: Achieving better medication adherence
}

Even when effective medical therapies are available and prescribed, patient adherence is an important factor in the actual clinical benefit experienced by patients. One postulated reason for poor adherence is patient out-of-pocket medication expenses. To examine the relationship between adherence and patient expenses, González López-Valcárcel and colleagues ${ }^{1}$ examined the effects of a change in costsharing in a population based study of over 10 thousand patients with an acute coronary event. A low income working population (no change in medication expenses) was compared with pensioners (a increase from no cost-sharing to 10\%) and a middle to high income working population (an increase in cost-sharing from $40 \%$ to $50 \%-60 \%)$. For low-cost essential medications, such as antiplatelet therapy and beta-blockers, the change in cost-sharing had no significant effect. However, for higher cost medications, such as statins, adherence temporarily decreased by about $8 \%$ in both pensioner and middle-high income populations, who experienced higher out-of-pocket costs (figure 1).

In the accompanying editorial, Mathews ${ }^{2}$ comments that these findings are aligned with previous studies showing poor medication adherence in acute coronary syndrome patients. In addition to out-of-pocket expenses, other factors such as health literacy and sociodemographic factors may contribute to non-adherence with prescribed medications. In addition, future studies should consider the effects of nonadherence on clinical outcomes; for example, the rate of coronary stent thrombosis with premature discontinuation of dual anti-platelet therapy. He concludes: 'Medication non-adherence is a widespread and pervasive problem across all disease states and one that spans the breadth of health delivery systems across the globe. It is time for the medication adherence paradigm to shift and be redefined in a place where patients and their providers share a mutual interest in achieving better adherence. Though patients may bear the final responsibility, it is incumbent on us as providers to create

Correspondence to Professor Catherine M Otto, Division of Cardiology, University of Washington, Box 356422, Seattle, WA 98195, USA; cmotto@uw.edu

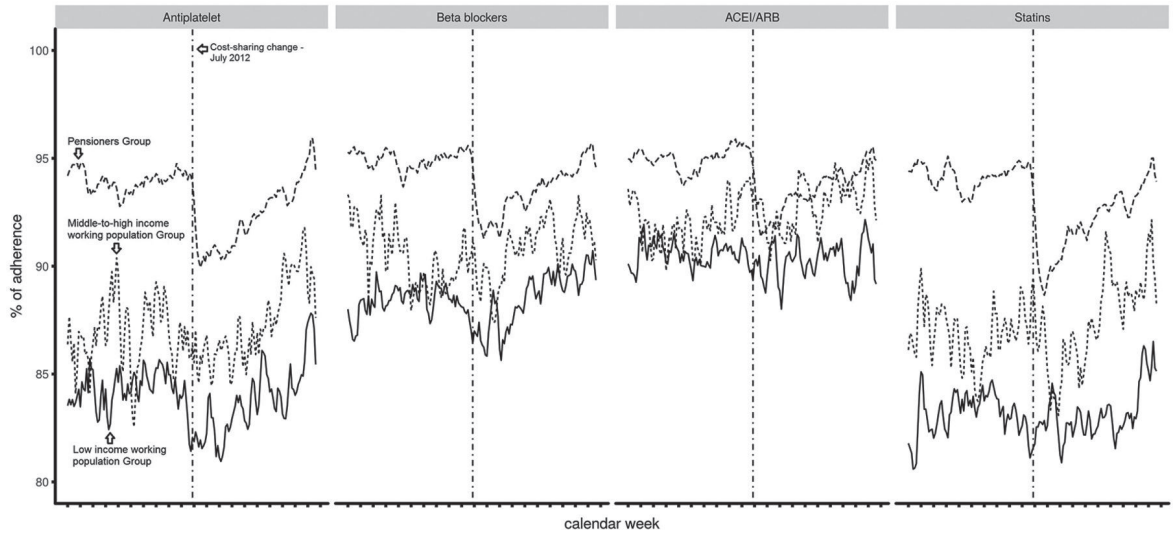

Figure 1 Weekly rates of adherence for the drugs considered for the three cohorts. ACEI, ACE inhibitor; ARB, angiotensin II receptor blocker. ${ }^{1}$ systems that place patients in the best position to advocate for their own health. Ultimately, solutions to meet this complex challenge need to be creative, pragmatic and scalable.'

Previous research on out-of-hospital cardiac arrest (OHCA) has focused on predictors of survival to hospital discharge with a paucity of data on factors predicting longer term survival. In this issue of Heart, Andrew and colleagues ${ }^{3}$ report a mean survival duration of about 12 years in 3449 patients who survived to hospital discharge after OHCA over a 14year period in Australia (figure 2). For the first year after OHCA, mortality was 5.6 times higher than expected but was similar to the general population 5 years post-OHCA. The only peri-arrest factor associated with long term survival was transport to a centre capable of percutaneous coronary intervention. Instead, in these patients who survived the initial OHCA event, long term survival was more related to post-discharge return to work and a favourable physical/functional recovery.

Kragholm and Torp-Pedersen ${ }^{4}$ suggest that these findings highlight our current knowledge gaps in survivors of OCHA. 'First, further insight into factors that are important for obtaining long survival are necessary to improve the outlook for these patients. Second, the marked result that survival quickly approached the background population should inspire to studies of whether there is early depletion of a high-risk group of patients such that few interventions are necessary after a certain time. Third, and finally, because a larger number of people survive out-of-hospital cardiac arrest and remain alive in long-term outcome assessments, further insights are needed into the longterm quality of life and other indicators of functional status.'

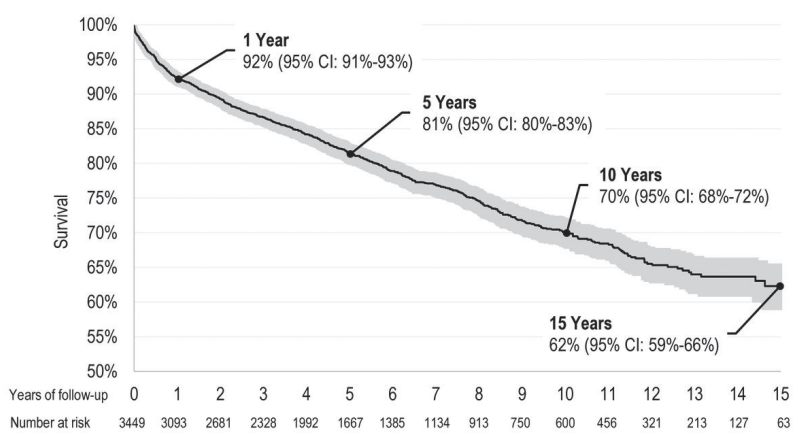

Figure 2 Kaplan-Meier survival estimates of out-of-hospital cardiac arrest survivors to hospital discharge. ${ }^{3}$ 
Device oriented composite events

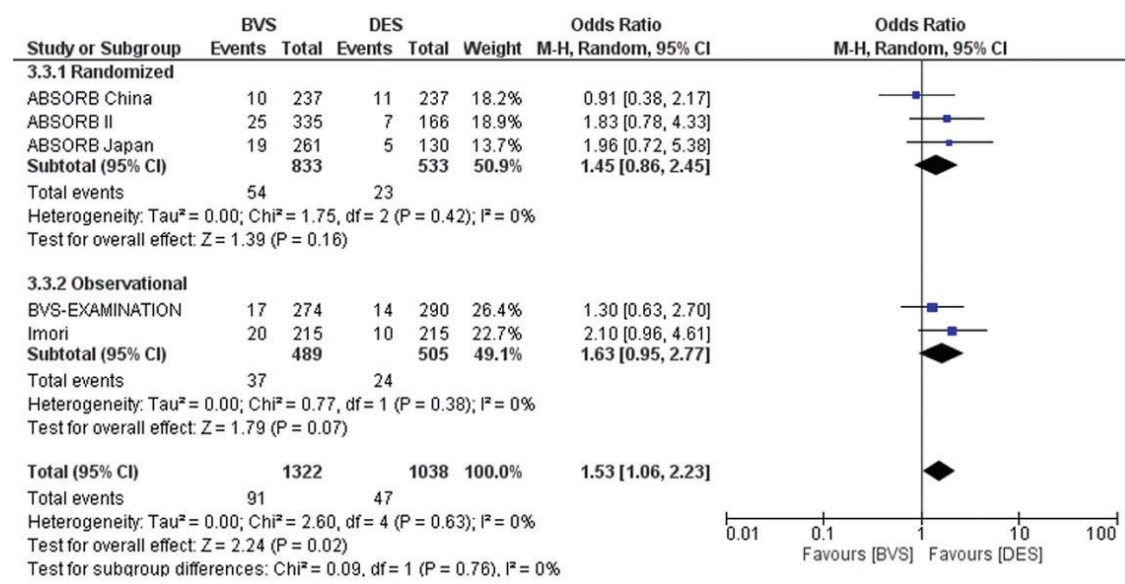

All-cause death

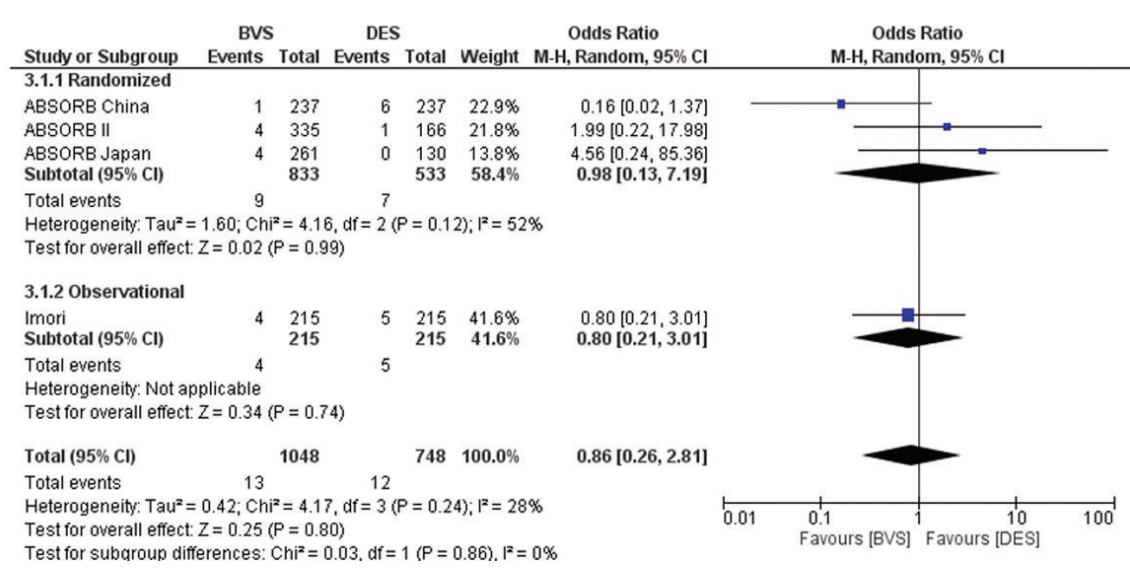

Figure 3 Long-term outcomes in coronary artery disease (CAD) with bioresorbable vascular scaffold (BVS) versus drug-eluting stent (DES). ${ }^{5}$

The best choice for a coronary stent in patients with coronary disease, ranging from silent ischemia to acute coronary syndrome, remains controversial. Nairooz and colleagues ${ }^{5}$ performed a meta-analysis outcomes at 2 years in studies comparing bioresorbable vascular scaffolds (BVS) to second generation drug eluting stents (DES). In 2360 patients from five trials, this meta-analysis showed that that the device-oriented composite endpoint (cardiac mortality and target vessel myocardial infarction or ischemia-driven revascularisation) was lower with DES compared with BVS, although there was no difference in all-cause mortality (figure 3).

In the accompanying editorial, Brugaletta and Sabate ${ }^{6}$ raise the concern

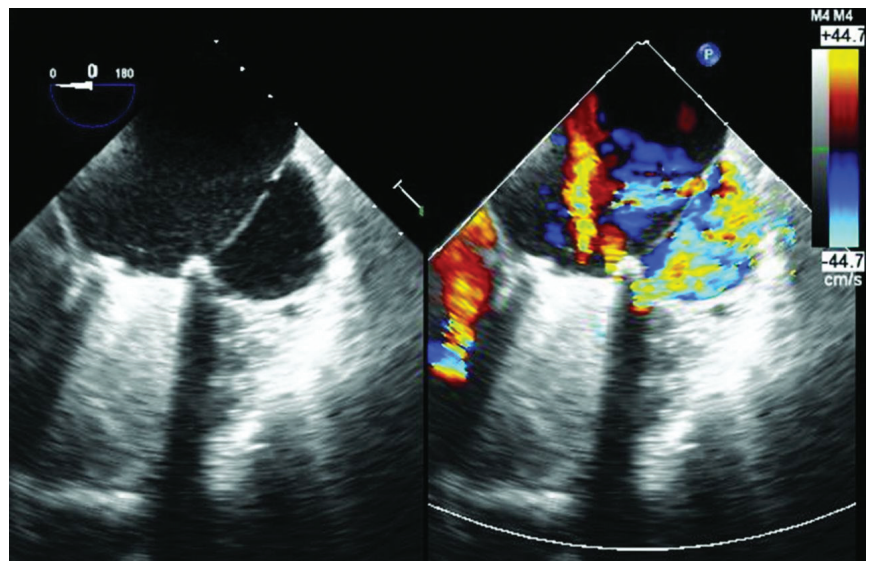

Figure 4

about BVS that 'the present meta-analysis represents a first attempt to understand the real incidence of scaffold thrombosis (ST) problem beyond 1 year after implantation, but many questions remain unanswered. As doctors having the safety of the patient as objective and as scientists with a close critical scrutiny on the clinical performance of these novel devices, we need to understand when ST may occur more frequently, which are the causes, how and if we can solve it.'

The Education in Heart article in this issue provides a summary of the literature, current guidelines and a practical algorithm for selecting the a novel oral anticoagulant in patients with atrial fibrillation. $^{7}$ The Image Challenge question ${ }^{8}$ asks you to make the diagnosis from an echocardiographic image (figure 4); the online videos are helpful in understanding the anatomy and physiology of this rare complication after mitral valve surgery.

Competing interests None declared.

Provenance and peer review Commissioned; internally peer reviewed.

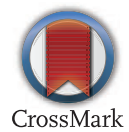

To cite Otto CM. Heart 2017;103:1057-1058.

Received 17 May 2017

Accepted 17 May 2017

Heart 2017:103:1057-1058

doi:10.1136/heartjnl-2017-311874

(C) Article author(s) (or their employer(s) unless otherwise stated in the text of the article) 2017. All rights reserved. No commercial use is permitted unless otherwise expressly granted.

\section{REFERENCES}

1 González López-Valcárcel B, Librero J, García-Sempere $A$, et al. Effect of cost sharing on adherence to evidence-based medications in patients with acute coronary syndrome. Heart 2017;103:1082-8.

2 Mathews R. Medication adherence: a moving target? Heart 2017:103:1059-60.

3 Andrew E, Nehme Z, Wolfe $R$, et al. Long-term survival following out-of-hospital cardiac arrest. Heart 2017;103:1104-10

4 Kragholm K, Torp-Pedersen C. Cardiac arrest survivors: short residual risk of death, long life expectancy. Heart 2017:103:1063-4.

5 Nairooz R, Saad M, Sardar P, et al. Two-year outcomes of bioresorbable vascular scaffold versus drug-eluting stents in coronary artery disease: a meta-analysis. Heart 2017;103:1096-103.

6 Brugaletta S, Sabate M. A longer look at trial evidence of bioresorbable vascular scaffolds. Heart 2017;103:1061-2.

7 Ghannam M, Chugh A. Indications and appropriate selection of novel oral anticoagulants in patients with atrial fibrillation. Heart 2017; 103:1129.

8 Kang Y, Huang R, He B. An abnormal chamber-like structure after mitral valve replacement. Heart 2017;103:1110. 